\section{Kontraindiziert bei Kuhmilcheiweißallergie}

\author{
Zu der im Referat „Schwieriger Milchersatz“ (Allergo Journal 2002; \\ 11: 358) vorgestellten Studie kommen aus der Schweiz kritische \\ Anmerkungen, was die Gabe von teilweise hydrolysierter Säuglings- \\ nahrung an Kinder mit nachgewiesener Kuhmilcheiweißallergie \\ anbetrifft.
}

$\mathrm{M}$ it Interesse haben wir das Referat zur Arbeit „Determination of allergenicity to three cow's milk hydrolysates and an amino acid-derived formula in children with cow's milk allergy" (Clin Exp Allergy 2002; 32: 74-9) von C. Caffarelli et al. gelesen. In der vorgestellten Studie wurden Kinder mit nachgewiesener Kuhmilcheiweißallergie mit verschiedenen Hydrolysatnahrungen inklusive einer auf Aminosäuren basierenden Nahrung therapeutisch getestet. Obwohl die Autoren selbst in der Einleitung angeben, dass teilweise hydrolysierte (moderat hydrolysierte) Säuglingsnahrung bei Kuhmilcheiweißallergie nicht geeignet ist, und dieses auch in ihren Studienergebnissen belegen, wurde ein solches Produkt (Humana HA, Mailand, Italien) verwendet. Auch laut europäischer Gesetzgebung [1] und der gemeinsamen Empfehlung von ESPGHAN und ESPACI [2] sind moderat hydrolysierte Säuglingsnahrungen bei Kuhmilcheiweißallergie kontraindiziert. Es ist daher unverständlich, warum Caffarelli et al. eine solche Nahrung in die orale Provokation einbezogen haben.

Laut EU-Gesetzgebung müssen Produkte mit moderaten Hydrolysaten einen entsprechenden Kontraindikationshinweis tragen, wonach sie bei Kuhmilcheiweissallergie nicht geeignet sind [1]. Zudem dürfen sie auch nur dann zur Prävention verwendet werden, wenn eine allergiepräventive Wirkung in prospektiven, kontrollierten klinischen Studien nachgewiesen werden konnte [1], was für die Nestlé-HA-Nahrungen (Beba HA, NAN HA, Nidina HA) zutrifft [3, $4,5,6,7,8]$. Es konnte nachgewiesen werden, dass sie bei nicht oder teilgestillten Säuglingen mit familiär erhöhtem Allergierisiko die allergischen Erstmani- festationen in den ersten 5 Jahren um die Hälfte reduzieren [3, 4, 5]. Im Tiermodell sind diese HA-Nahrungen in der Lage, eine orale Toleranz auf das verwendete Ausgangsprotein (Molkeneiweiß) $\mathrm{zu}$ induzieren, was das eigentliche Ziel der Allergieprävention ist $[9,10]$. Weiterhin führen sie bei nicht oder teilgestillten Säuglingen zu einer besseren Nahrungsverträglichkeit und rufen in den ersten 2 Lebensjahren signifikant weniger Hautprobleme hervor als herkömmliche Säuglingsnahrungen [11, $12,13]$.

Wir möchten festhalten, dass moderat hydrolysierte Säuglingsnahrungen in der Behandlung von Kuhmilcheiweissallergie kontraindiziert sind. Hingegen sind sie die derzeit beste Alternative für nicht oder teilgestillte Säuglinge, ungeachtet, ob ein erblich erhöhtes Allergierisiko bekannt ist oder nicht $[14,15,16]$.

\section{Dr. Bianca-Maria Exl-Preysch, PhD Prof. Dr. Wolf Endres Nestlé Nutrition, Nestec Ltd Case Postale 352 CH-1800 Vevey Schweiz}

\section{Literatur}

1. (ECC) European Communities Commission Directive amending Directive 91/321/EEC on infant formulae and follow on formulae; 11/5769/93-EN-Rev 4; adopted 16.2.96. Official Journal of the European Communities. No. L49/39, 1996

2. Joint statement of ESPACI and ESPGHAN (Höst A, Koletzko B, Dreborg S, Muraro A, Wahn U, Aggett P, Bresson J-L, Hernell O, Lafeber H, Michaelson KF, Micheli J-L, Rigo J, Weaver L, Heymans H, Strobel S, Vandenplas Y). Dietary products used in infants for treatment and prevention of food allergy. Arch Dis Child 1999; 81: 80-4

3. Vandenplas $Y$, Hauser B, van den Borre $C$, Clybouw O Mahler T, Hachimi-Idrissi S,
Deraeve L, Malfroot A, Dab I. The long-term effect of a partial whey hydrolysate formula on the prophylaxis of atopic disease. Eur J Pediatr 1995a; 154: 488-94

4. Chandra RK. Five-year follow-up of highrisk infants with family history of allergy who were exclusively breast-fed or fed partial hydrolysate, soy, and conventional cow's milk formulae. J Ped Gastroent Nutr 1997; 24: 380-8

5. Baumgartner $M$, Brown CA, Exi B- $M$, Secretin $M-C$, van't Hof $M$, Haschke F. Controlled trials investigating the use of one partially hydrolyzed whey formula for dietary prevention of atopic manifestations until 60 months of age: an overview using metaanalytical techniques. Nutr Res 1998; 18: 1425-42

6. von Berg A, Koletzko S, Grübl A, Schoetzau A, Wichmann HE, Bauer CP, Reinhardt D, Berdel D. GINI - German International Nutrition Intervention Programme. First Preliminary Results at the age of 12 months. EAACI Berlin und ESPGHAN Genf, 2001

7. Friedrich F. Erste Ergebnisse der GINI-Studie. Pädiatrische Allergologie 2001; 2: 12-3

8. EAACI, Section on Pediatrics. Annual Symposium „Prevention of Food Allergy“. Book of Abstracts. Abano Terme, Padua, February 2002

9. Fritsché R, Pahud JJ, Pecquet S, Pfeifer A. Induction of systemic immunological tolerance to beta-lactoglobulin by oral administration of a whey protein hydrolysate. J Allergy Clin Immunol 1997; 100: 266-73

10. Peyquet S, Bovetto L, Maynard F, Fritsché R. Peptides obtained by tryptic hydrolysis of bovine beta-lactoglobulin induce specific oral tolerance in mice. J Allergy Clin Immun 2000: 514-21

11. ExI BM, Deland U, Secretin MC, Preysch U, Wall $M$, Shmerling DH. Improved general health status in an unselected infant population following an allergen reduced dietary intervention programme: The ZUFFstudy-programme. Part 1: study design and 6-month nutritional behaviour. Eur J Nutrition 2000; 39: 89-102

12. ExI BM, Deland U, Secretin MC, Preysch U, Wall $M$, Shmerling $\mathrm{DH}$. Improved general health status in an unselected infant population following an allergen reduced dietary intervention programme: The ZUFF study programme. Part 11: infant growth and health status to age 6 months. Eur J Nutrition 2000; 39: 145-56

13. ExI BM, Deland U, Secretin MC, Preysch U, Wall $\mathrm{M}$, Shmerling $\mathrm{DH}$. Improved general health status in an unselected infant population following an allergen reduced dietary intervention programme: The ZUFF study programme. Part IV: infant growth and health status to age 2 years. Eur J Nutrition 2003; in press. Abstract: J Pediatric Gastroenterology and Nutrition 2000; 31: abstract 100

14. Vandenplas Y. Primary and secondary prevention of atopic diseases in children. Singapore Paediatr J 1999; 41: 192-205

15. ExI BM. A review of recent developments in the use of moderately hydrolysed whey formulae in infant nutrition. Nutrition Research 2001; 21: 355-79

16. ExI BM, Fritsché R. Cow's milk protein allergy and possible means for its prevention. Int J Nutrition 2001; 17: 642-51 